\title{
Theta synchronization over occipito-temporal cortices during visual perception of body parts
}

\author{
Quentin Moreau, ${ }^{1,2}$ (D) Enea F. Pavone, ${ }^{2,3}$ Salvatore M. Aglioti ${ }^{1,2}$ and Matteo Candidi, ${ }^{1,2}$ \\ ${ }^{1}$ SCNLab, Department of Psychology, Sapienza University of Rome, Via dei Marsi, 78, 00185 Rome, Italy \\ ${ }^{2}$ IRCCS Fondazione Santa Lucia, Rome, Italy \\ ${ }^{3}$ BrainTrends Itd Applied Neuroscience, Rome, Italy
}

Keywords: body parts visual perception, EEG, theta band, time-frequency

\begin{abstract}
Categorical clustering in the visual system is thought to have evolved as a function of intrinsic (intra-areal) and extrinsic (interareal) connectivity and experience. In the visual system, the extrastriate body area (EBA), an occipito-temporal region, responds to full body and body part images under the organizational principle of their functional/semantic meaning. Although frequency-specific modulations of neural activity associated with perceptive and cognitive functions are increasingly attracting the interest of neurophysiologists and cognitive neuroscientists, perceiving single body parts with different functional meaning and full body images induces time-frequency modulations over occipito-temporal electrodes are yet to be described. Here, we studied this issue by measuring EEG in participants who passively observed fingers, hands, arms and faceless full body images with four control plant stimuli, each bearing hierarchical analogy with the body stimuli. We confirmed that occipito-temporal electrodes (compatible with the location of EBA) show a larger event-related potential (ERP, N190) for body-related images. Furthermore, we identified a body part-specific (i.e. selective for hands and arms) theta event-related synchronization increase under the same electrodes. This frequency modulation associated with the perception of body effectors over occipitotemporal cortices is in line with recent findings of categorical organization of neural responses to human effectors in the visual system.
\end{abstract}

\section{Introduction}

The body of our conspecific conveys socially relevant information, and the brain dedicates many resources to processing this type of stimulus. Research on the neural correlates of body perception provides evidence of an involvement of high-level visual areas specifically dedicated to processing body images. Yet, whether different neural computations may support the processing of different body parts remains unclear. Imaging (fMRI) studies (Downing et al., 2001; Peelen \& Downing, 2005) have shown that two bilateral regions of the visual system [fusiform body a(FBA) and the extrastriate body area (EBA)] selectively respond to the observation of bodies and body parts compared to non-body stimuli. A causal role for the activity of the EBA for processing body images has been provided by brain stimulation and lesions studies (Urgesi et al., 2004; Moro et al., 2008). Transcranial magnetic stimulation (TMS)

Correspondence: Quentin Moreau and Dr Matteo Candidi, ${ }^{1}$ SCNLab, as above. E-mails: quentin.moreau@uniroma1.it and matteo.candidi@uniroma1.it

Received 28 June 2017, revised 21 November 2017, accepted 21 November 2017

Edited by Gregor Thut

Reviewed by Marius Peelen, University of Trento, Italy; Jim Herring, University of Amsterdam, Netherlands

The associated peer review process communications can be found in the online version of this article. revealed that modulating the EBA activity $\sim 150 \mathrm{~ms}$ after image presentation impairs perceptual discrimination between different full body images and different single body part images (Urgesi et al., 2004, 2007; Pitcher et al., 2009, 2012).

Recent studies have shown that subclusters within the EBA territory exhibit a body-part selectivity with segregated voxels showing greater responses to hands, arms or torso images (Schwarzlose et al., 2005; Bracci et al., 2010; Orlov et al., 2010; Weiner \& GrillSpector, 2010). Moreover, body parts that are semantically coded as motor effectors [i.e. hands (Bracci et al., 2012), arms and legs (Bracci et al., 2015)] are represented in neighbouring regions within the occipito-temporal cortex, suggesting that the action-relatedness of a given body part is a fundamental organizational principle in the occipito-temporal pole (Bracci \& Peelen, 2013; Bracci et al., 2015; Lingnau \& Downing, 2015). These findings contribute to the debate concerning whether the functional role of EBA (and the FBA) is to code for single body parts or for their spatial arrangement in a full body shape (i.e. part-based vs. holistic body representation; Taylor et al., 2007; see also Brandman \& Yovel, 2016).

Adding to the functional imaging and brain stimulation evidence, EEG (Thierry et al., 2006) and intracranial studies (Pourtois et al., 2007) have characterized the timing of the neural response to body images by identifying a body-specific event-related potential (ERP) that appears as a cortical negative potential peaking around $200 \mathrm{~ms}$ after stimulus onset (i.e. body-N190). This ERP is identified 
bilaterally over the occipito-temporal electrodes (PO7, PO8, P7, P8) and is assumed to be generated by the EBA, as suggested by source localization analysis (Thierry et al., 2006; Giabbiconi et al., 2016) and intracranial recordings (Pourtois et al., 2007).

Although EEG studies have described an early neural response to body images in the time domain, there have not been any studies testing frequency-specific modulations associated with visual processing of different body parts and full body images. Interest in frequency analysis is gaining momentum as frequency-specific activity is thought to reflect different areal and interareal processing mechanisms (Buzsaki \& Draguhn, 2004; Fries, 2009, 2015; Wang, 2010). Importantly, categorical clustering in the visual system may result from a combination of anatomical and functional constraints such as the experience with, and ecological relevance of, specific stimuli as well as the intrinsic and extrinsic connectivity within the visual system and between the visual system and other cortical regions (Kravitz et al., 2013). Describing local oscillatory neural responses to stimuli of different categories and subcategories may represent an indication of the different network mechanisms supporting their processing and their distinct neural fate (Peelen \& Caramazza, 2010).

In this study, besides replicating earlier findings highlighting the body category-specific N190 amplitude modulation (Pourtois et al., 2007; Taylor et al., 2007), we performed Event-Related (De)Synchronization (ERD/ERS) analyses (Pfurtscheller \& Lopes da Silva, 1999; Pfurtscheller, 2001) to describe band-specific changes in poststimulus EEG power associated with the processing of body-related visual stimuli. Because several studies have associated the visually evoked P1-N1 complex to theta $(4-7 \mathrm{~Hz})$ and alpha $(8-13 \mathrm{~Hz})$ band modulations (Klimesch et al., 2004; Gruber et al., 2005), we also studied power modulations (i.e. Whole, Evoked and Induced Power) in these frequency ranges in the P1-N1 time window (i.e. 75-225 ms).

To summarize, we compared two different categories of stimuli (Bodies vs. Plants) at four different hierarchical levels (finger/leaf, hand/cluster, arm/branch, body/tree). On the one hand, we replicated previous findings in the time domain (i.e. higher N190 amplitude for body images compared to control ones) while, on the other hand, we extended the characterization of this response to body part images in the occipito-temporal cortex by describing a selective increase in theta Whole and Evoked power associated with the observation of specific body effectors (i.e. hand and arm).

\section{Materials and methods}

\section{Participants}

Sixteen individuals (five male, 11 female, mean age of $27.1 \pm 4.1$ ) took part in the experiment. All participants were right-handed with normal or corrected-to-normal vision and gave written consent prior to the experiment. Participants were naive as to the aim of the experiment at the outset and were informed of the purpose of the study only after all the experimental procedures were completed. All participants were paid $7 € / \mathrm{h}$. The experimental procedures were approved by the Ethical Committee of the Fondazione Santa Lucia (Rome, Italy), and the study was performed in accordance with the 1964 Declaration of Helsinki.

\section{Stimuli}

All stimuli were used in a previous EEG study by Taylor et al. (2010). The body stimuli (Bodies category) comprised a total of 32 grayscale images divided into four groups according to their hierarchy: (1) male or female headless full body, (2) single arm, (3) single hand and (4) single finger. Control stimuli (Plants category) comprised a total of 32 grayscale images also divided into four groups: (1) whole tree, (2) branch, (3) leaf cluster and (4) individual leaf. All images were scaled to $400 \times 400$ pixels at a resolution of 72 dpi on a 'Netscape grey' background (RGB, 192 : 192 : 192). Example stimuli are presented in Fig. 1.

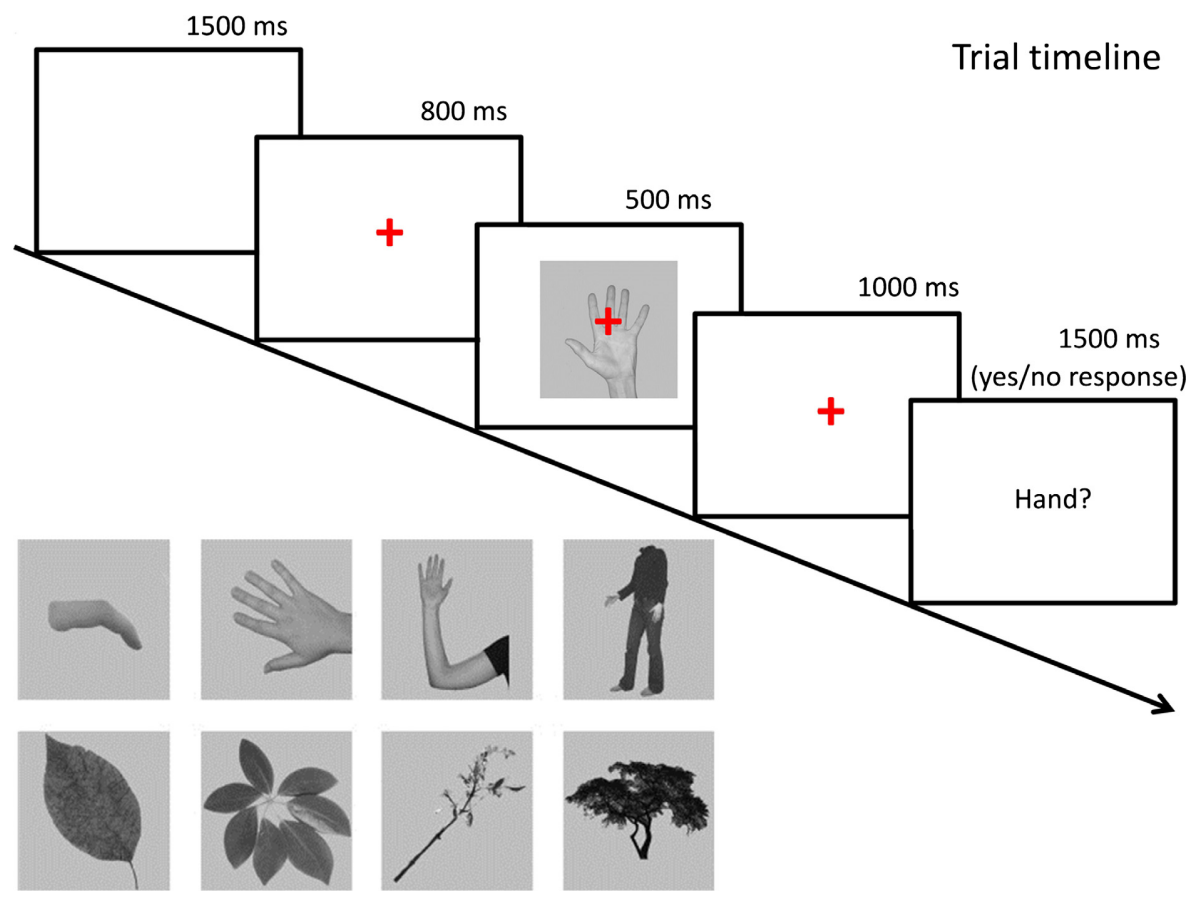

FIG. 1. Trial timeline and examples of stimuli (adapted from Taylor et al., 2010). 


\section{Procedure}

Each trial comprised of a red fixation cross that appeared $800 \mathrm{~ms}$ before the target stimulus. Target images were shown for $500 \mathrm{~ms}$ at the centre of the screen. To avoid EEG artefacts from the appearance and disappearance of the fixation cross, the fixation cross remained during and $1500 \mathrm{~ms}$ after the disappearance of the target stimuli, followed by a blank screen for $1500 \mathrm{~ms}$. Stimuli were viewed at a distance of approximately $60 \mathrm{~cm}$. To maintain vigilance over the stimuli, on random trials (20\% of the trials), participants were asked to respond by pressing one of two buttons with their left index and middle finger to indicate whether the previous stimulus was a leaf, leaf cluster, branch, tree, finger, hand, arm or body image (e.g. Leaf? Yes/No). Stimulus presentation and synchronization with the EEG system were controlled via E-PRIME software (Psychology Software Tools Inc., Pittsburgh, PA, USA). The experiment consisted of six blocks with 64 trials each, where Bodies and Plants stimuli were presented randomly. The total number of trials was 384 (48 trials for each of the eight different conditions).

\section{EEG-recordings}

EEG signals were recorded and amplified using a Neuroscan SynAmps RT amplifiers system (Compumedics Limited, Melbourne, Australia) and acquired from 60 tin scalp electrodes embedded in a fabric cap (Electro-Cap International, Eaton, $\mathrm{OH}$ ), arranged according to the 10-10 system. The EEG was recorded from the following channels: Fp1, Fpz, Fp2, AF3, AF4, F7, F5, F3, F1, Fz, F2, F4, F6, F8, FC5, FC3, FC1, FCz, FC2, FC4, FC6, T7, C5, C3, C1, Cz, C2, C4, C6, T8, TP7, CP5, CP3, CP1, CPz, CP2, CP4, CP6, TP8, P7, P5, P3, P1, Pz, P2, P4, P6, P8, PO7, PO3, AF7, POz, AF8, PO4, $\mathrm{PO} 8, \mathrm{O} 1, \mathrm{Oz}, \mathrm{O} 2, \mathrm{FT} 7$ and FT8. Horizontal electrooculogram (HEOG) was recorded bipolarly from electrodes placed on the outer canthi of each eye, and signals from the left earlobe were also recorded. All electrodes were physically referenced to an electrode placed on the right earlobe and were algebraically re-referenced offline to the average of both earlobe electrodes. Impedance was kept below $5 \mathrm{~K} \Omega$ for all electrodes for the whole duration of the experiment, amplifier hardware band-pass filter was $0.01-200 \mathrm{~Hz}$, and sampling rate was $1000 \mathrm{~Hz}$. To remove the eye blinks or other artefacts, EEG and horizontal electro-oculogram were processed in two separate steps. Data were then downsampled at $500 \mathrm{~Hz}$ before a blind source separation method was applied, using Independent Component Analysis (ICA) (Jung et al., 2000) implemented in the MATLAB toolbox EEGLab (Delorme \& Makeig, 2004) to remove from the EEG any components related to eye movements. Trials showing amplifier blocking, residual eye blink or other types of artefacts were then excluded from the analysis manually. The artefact rejection procedure (over all the 16 participants) led to $11.85 \%$ of the trials being rejected (final average of trials per condition $=42.31$, std $=3.13$ ).

\section{Analyses}

\section{Event-related potentials}

Analyses of P1-N1 components were carried out with BRAIN VISION ANALYZER 1.05 software (Brain-Products, GmbH). Prior to ERP averaging for each condition, EEG epochs over the range -200 to $+1000 \mathrm{~ms}$ relative to stimulus onset were baseline corrected with reference to the pre-stimulus signal of $200 \mathrm{~ms}$. P1 and N1 mean amplitudes were computed using time windows ranging from 80 to $150 \mathrm{~ms}$ and 150 to $220 \mathrm{~ms}$, respectively. These time windows were centred on the grand average peak latency of P1 and N1 values (116 ms for P1 and $189 \mathrm{~ms}$ for N1, respectively).

\section{Clusters of electrodes}

In order to compare several areas of the occipital, parieto-occipital and occipito-temporal cortices, three different clusters of electrodes were computed. The different clusters of electrodes are as follows: (1) the extrastriate body area cluster (i.e. EBA) (PO8, P8, PO7 and P7) (Thierry et al., 2006; Pourtois et al., 2007; Taylor et al., 2010); (2) the occipital cluster (i.e. OCC) (O1 and O2); (3) the parieto-occipital cluster (i.e. PO) (PO4, P6, PO3 and P5).

\section{Time-frequency analysis}

\section{Whole, evoked and induced power}

Time-frequency analyses were performed using the FieldTrip routines (Donders Institute, Nijmegen; Oostenveld et al., 2010) in MATLAB (The MathWorks, Inc.). The EEG time series were obtained by segmenting the signal into epochs of $1500 \mathrm{~ms}$ length (from $500 \mathrm{~ms}$ before to $1000 \mathrm{~ms}$ after the presentation of the stimulus) and were band-pass filtered $(0.1-100 \mathrm{~Hz})$. Each epoch was transformed in the frequency domain and multiplied with the fast Fourier transformation (FFT) power spectrum of a set of complex Morlet wavelets using $50 \mathrm{~ms}$ time bins in a -500 to $1000 \mathrm{~ms}$ peristimulus window. A width of four provided an adequate trade-off between temporal and frequency resolution (Cavanagh et al., 2012).

Time frequency analysis on single trials resulted in the computation of the Whole power, containing both phase-locked signal (i.e. Evoked power) and non phase-locked signal (i.e. Induced power). Then, Evoked power was calculated from averaged ERPs (for each subject and condition), and Induced power was identified by subtracting Evoked from Whole power for each single trial. Estimated frequency band results for Whole and Induced Power were displayed as event-related desynchronization/synchronization (ERD/ ERS) with respect to a baseline between -500 and $-100 \mathrm{~ms}$ before presentation of the stimulus. Relative change in Evoked power was determined by subtracting the baseline-corrected Induced power from the baseline-corrected Whole power. Indeed, baseline signal in Evoked power is very small due to averaging, and computing ERD/ ERS on Evoked activity would result in meaningless results (Hajihosseini \& Holroyd, 2013;.). ERD and ERS represent a decrease or increase in synchrony of the recorded neuronal population (Pfurtscheller \& Lopes da Silva, 1999). Positive and negative ERD/ ERS values index synchronization and desynchronization with respect to a given reference interval. The formula used to compute event-related desynchronization/synchronization was as follows:

$$
\operatorname{ERD} / \operatorname{ERS}(t, f)=\frac{\mathrm{E}(t, f)-\mathrm{E}_{\mathrm{ref}}(t, f)}{\mathrm{E}_{\mathrm{ref}}(t, f)}
$$

where $\mathrm{E}(t, f)$ represents the spectrum at a given $t$ (time) and $f$ (frequency) and $\mathrm{E}_{\mathrm{ref}}(t, f)$ is the mean power of the reference interval. For each experimental condition, ERD/ERS were extracted from 75 to $225 \mathrm{~ms}$ (to include both $\mathrm{P} 1$ and $\mathrm{N} 1$ peaks) on EBA electrodes (P08, P07, P8, P7), at both Theta $(4-7 \mathrm{~Hz})$ and Alpha $(8-13 \mathrm{~Hz})$.

\section{Statistics}

Statistical analyses on ERPs (i.e. P1, N1 mean amplitudes) and ERD/ERS were performed with statsoft statistica 8 software. 
General linear model (GLM) and the Greenhouse-Geisser correction for non-sphericity were applied when appropriate (Keselman \& Rogan, 1980). P1 and N1 mean amplitudes were analysed through two separated $3 \times 2 \times 4$ within-subject repeated measures AVOVAs with factors Electrodes cluster (OCC, EBA, PO), Category (Bodies, Plants), Hierarchy (finger/leaf, hand/leaf cluster, Arm/Branch, Body/Tree). Time-frequency indexes (i.e. Whole and Evoked Theta ERD/ERS) were analysed each at EBA by a $2 \times 4$ within subject repeated measures ANOvAs with factors Category (Bodies, Plants) and Hierarchy (finger/leaf, hand/leaf cluster, arm/ branch, body/tree). Post hoc comparisons were performed using the Newman-Keuls correction for multiple comparisons.

\section{Results}

\section{Accuracy}

Mean overall recognition performance was $91.83 \pm 2.9 \%$ (mean $\pm \mathrm{SEM})$.

\section{Time domain - ERPs}

\section{P1 mean amplitude}

The $3 \times 2 \times 4$ (Cluster of Electrodes $\times$ Category $\times$ Hierarchy) ANOVA showed that the factor Category did not reach statistical significance as a main effect $(P=0.132)$ nor did it interact with any other factor (all $P \mathrm{~s}>0.300$ ), thus showing that P1 mean amplitude was not affected by the content of the stimuli. The analysis highlighted a significant main effect of Electrodes $\left(F_{2,30}=4.809, P=0.032\right)$, indicating higher mean amplitudes at EBA compared to PO $(P=0.012$, all other $P \mathrm{~s}>0.09)$. No other main effect was significant (all $P$ s $>0.338$ ). The only interaction that turned out to be significant was that which occurred between Electrodes and Hierarchy $\left(F_{6,90}=6.470, P<0.001\right)$. Post hoc test indicated that the hierarchy factor had a different impact on P1 mean amplitude at EBA and PO compared to OCC. Indeed: (1) in EBA and PO, only the third hierarchical level (i.e. arm/branch) induced lower P1 amplitudes compared to the other levels $(P s<0.03)$ and (2) conversely, in OCC, each hierarchical level differed from the others (all $P$ s $<0.001$ ) except the second (i.e. hand/leaf cluster) vs. third (i.e. arm/branch) hierarchical level $(P=0.390)$.

\section{N1 mean amplitude}

The $3 \times 2 \times 4$ (Cluster of Electrodes $\times$ Category $\times$ Hierarchy) ANOVA on N1 mean amplitude revealed that the triple Electrodes $\times$ Category $\times$ Hierarchy interaction reached significance $\left(F_{6,90}=5.368, P<0.001\right)$. Post hoc comparisons showed that: (1) all body stimuli induced larger $\mathrm{N} 1$ mean amplitudes than plant images in EBA electrodes (all $P_{\mathrm{s}}<0.001$ ) (see Figs 2 and 3); (2) only full bodies induced larger amplitudes than trees over the OCC $(P<0.001)$; (3) finger, hand and full bodies induced larger mean amplitudes compared to leaves, leaf clusters and trees over the PO (all $P \mathrm{~s}<0.001$ ).

The factor Category (Bodies vs. Plants) reached statistical significance $\left(F_{1,15}=9.159, P=0.009\right)$ and showed that Bodies induced a significantly greater $\mathrm{N} 1$ mean amplitude compared to plants overall. The factor Hierarchy reached significance $\left(F_{3,45}=8.470\right.$, $P<0.001$ ) with larger N1 for level 2 (hands/leaf cluster) than level 4 (body/tree) $(P=0.004)$, and larger N1 for level 3 (arm/branch) than level 1 (finger/leaf) $(P=0.016)$ and four (body/tree) $(P<0.001)$. It was also found that the Electrodes $\times$ Hierarchy interaction was significant $\left(F_{6,90}=12.691, P<0.001\right)$, with levels 2 (hands/leaf cluster) and 3 (arm/branch) inducing a larger N1 than level 4 (body/tree) in all Electrodes (all $P$ s $<0.001$ ), level 1 (finger/ leaf) inducing smaller N1 than 2 (hands/leaf cluster), 3 (arm/branch) and 4 (body/tree) in OCC $(P \mathrm{~s}<0.001)$, smaller than 2 (hands/leaf cluster), 3 (arm/branch) and 4 (body/tree) in EBA ( $P$ s $<0.001)$, and smaller only than 3 (arm/branch) and 4 (body/tree) in $\mathrm{PO}$ $(P<0.001)$.

Thus, by replicating previous studies (Thierry et al., 2006; Pourtois et al., 2007; Taylor et al., 2010), we found it was only at EBA cluster that all body stimuli induced larger N1 mean amplitudes when compared to plant images as a whole category, and this also applied to each hierarchical level. Then, we tested the hypothesis that this EBA response to body-part stimuli is also characterized by band-specific modulations.

\section{Time-frequency analyses}

\section{Whole power}

Alpha $(8-13 \mathrm{~Hz})$ on EBA cluster. The $2 \times 4$ ANOvA with Category (Body vs. Plants) $\times$ Hierarchy (finger/leaf, hand/leaf cluster, arm/ branch, body/tree) revealed a main effect of Hierarchy $\left(F_{3,45}=3.978, P=0.016\right)$. Post hoc tests indicated that the second level (hand and leaf cluster) induced larger alpha ERS than the other hierarchical levels $\left(P_{\mathrm{s}}<0.01\right)$ (all other $\left.P \mathrm{~s}>0.12\right)$.

Theta $(4-7 \mathrm{~Hz}$ ) on EBA cluster. The $2 \times 4$ ANOva with Category (Body vs. Plants) $\times$ Hierarchy (finger/leaf, hand/leaf cluster, arm/ branch, body/tree) revealed a significant second level Category $\times$ Hierarchy interaction $\left(F_{3,45}=3.978, P=0.013\right)$ (Fig. 4). Post hoc tests indicated that the hand and arm images induced larger theta ERS than leaf cluster and branch images (both $P$ s $<0.015)$. Furthermore, hand and arm images induced higher theta ERS than finger $(P<0.001)$ and body images $(P<0.002)$.

Overall, whole power ERD/ERS analyses over EBA showed a specific theta ERS increase for hand and arm images when compared with all other body parts (i.e. fingers and bodies) as well as with all control non-body stimuli.

\section{Evoked power}

Alpha $(8-13 \mathrm{~Hz})$ on EBA cluster. The $2 \times 4$ ANOva with Category (Body vs. Plants) $\times$ Hierarchy (finger/leaf, hand/leaf cluster, arm/ branch, body/tree) revealed neither no significant main effect nor interaction (all $P \mathrm{~s}>0.055$ ).

Theta $(4-7 \mathrm{~Hz})$ on EBA cluster. The $2 \times 4$ ANova with Category (Body vs. Plants) $\times$ Hierarchy (finger/leaf, hand/leaf cluster, arm/ branch, body/tree) revealed a significant second level Category $\times$ Hierarchy interaction $\left(F_{3,45}=6.061, P=0.011\right)$ (Fig. 5). Post hoc tests indicated that the hand and arm images induced larger theta ERS than leaf cluster and branch images (both $P \mathrm{~s}<0.007$ ). Furthermore, hand and arm images induced higher theta ERS than finger $(P<0.001)$ and body images $(P<0.001)$.

Overall, Evoked power analyses over EBA showed a specific theta synchronization increase for hand and arm images when compared with all other body parts (i.e. fingers and bodies) as well as with all control non-body stimuli. 
A

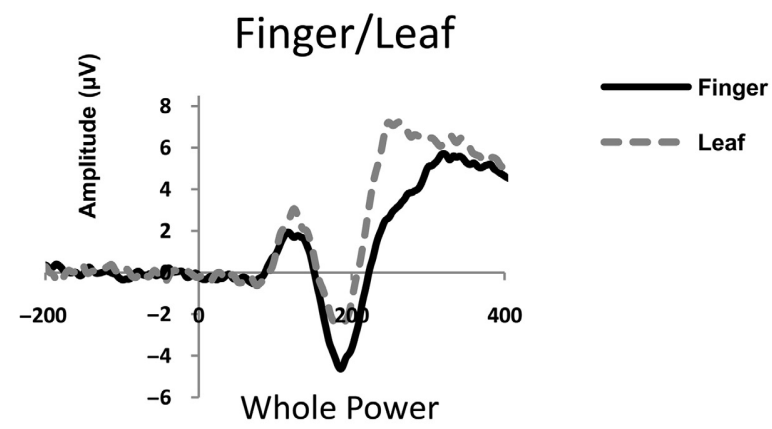

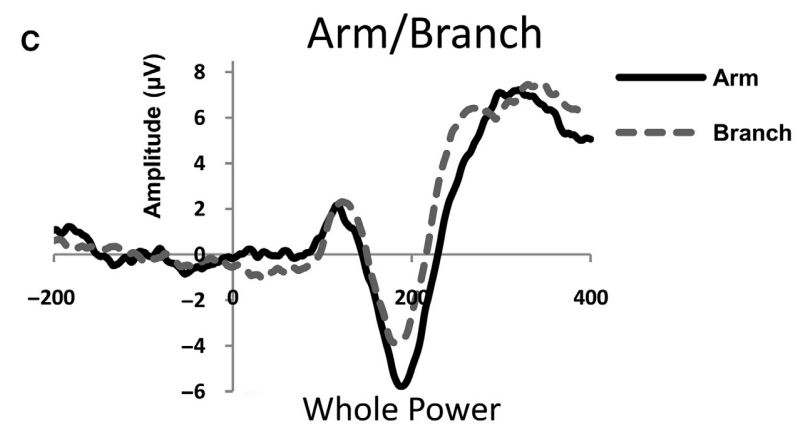
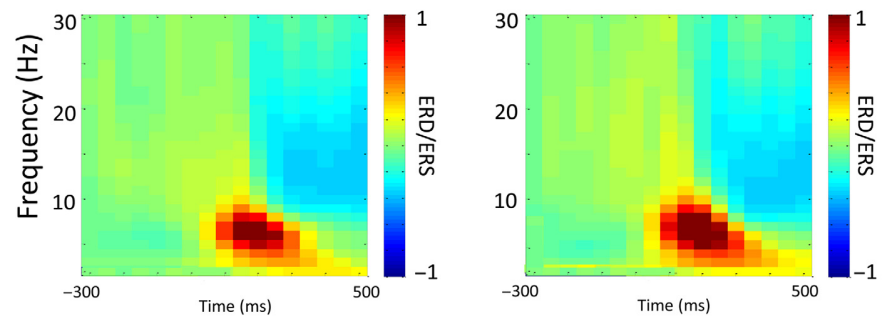

Evoked Power
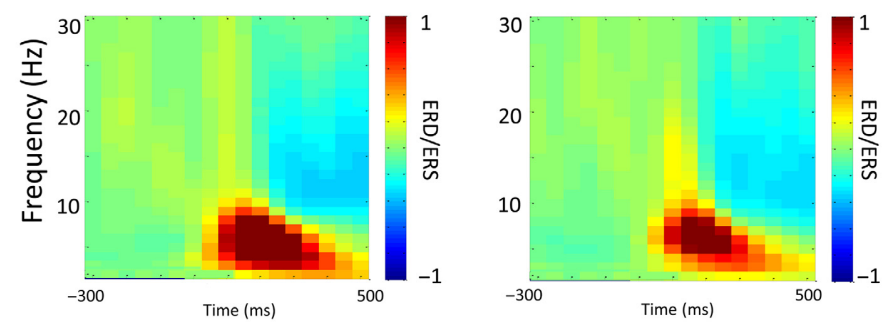

Evoked Power
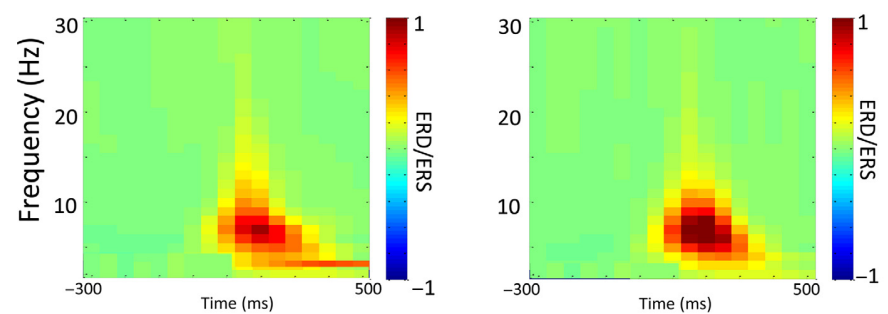

B
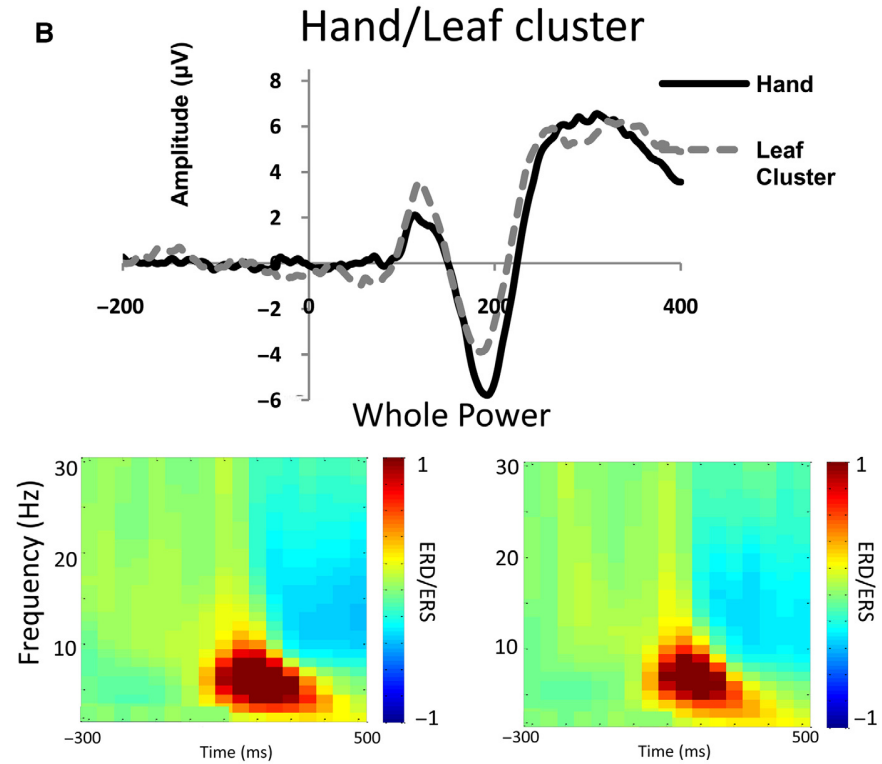

Evoked Power
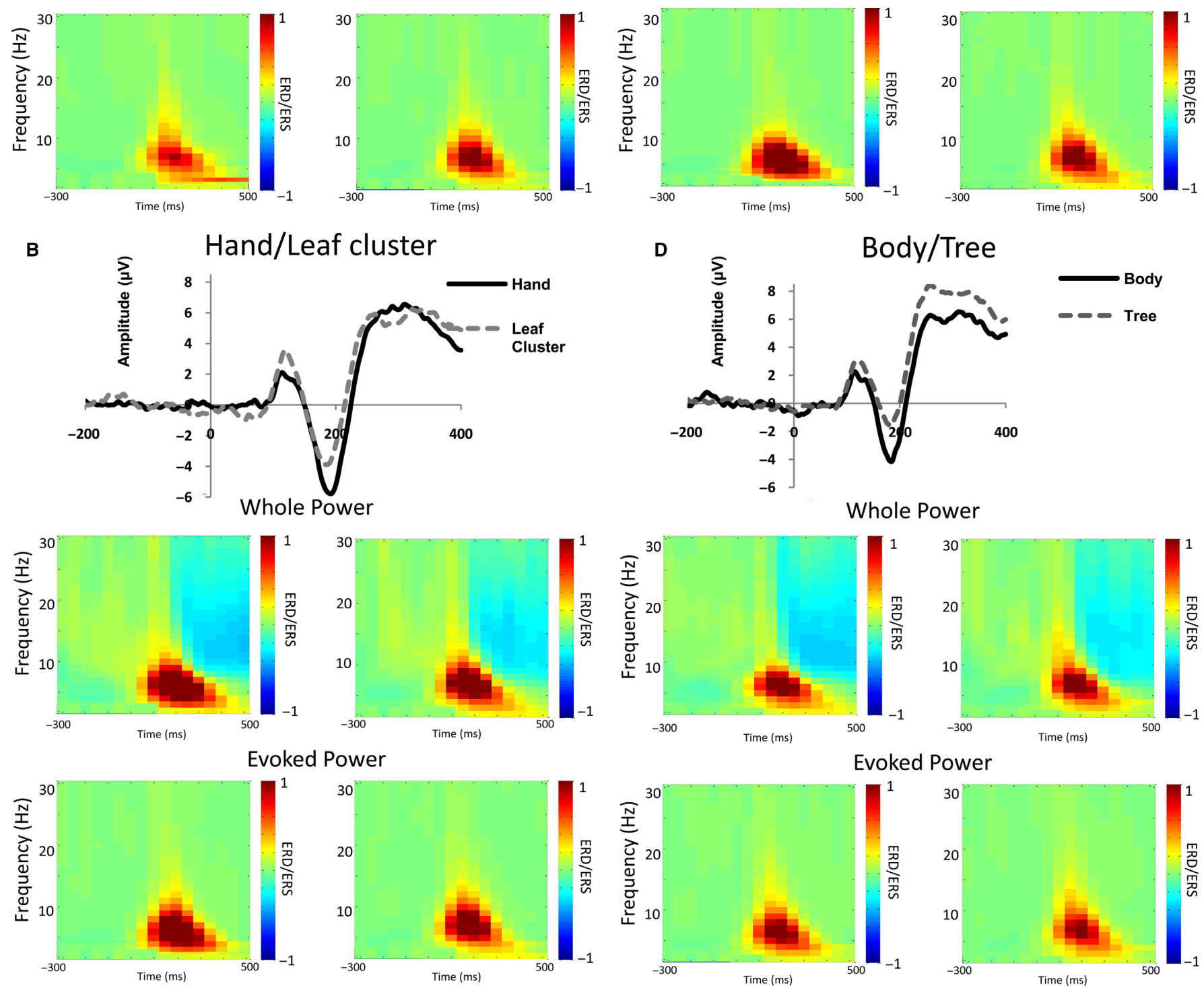

D

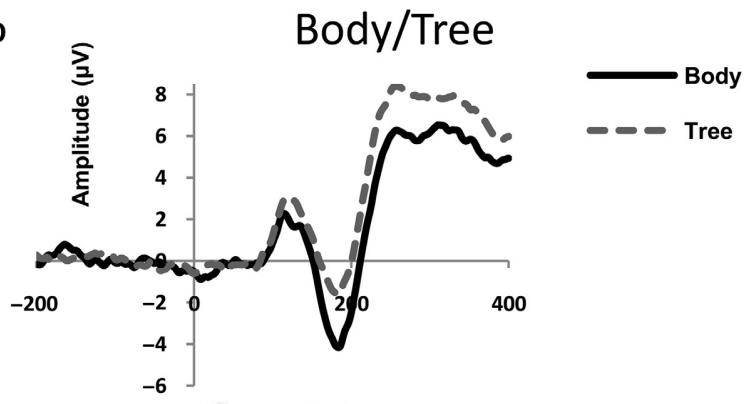

Whole Power
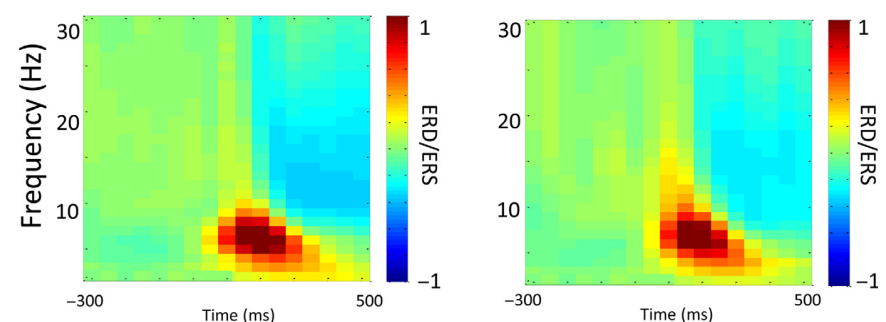

Evoked Power
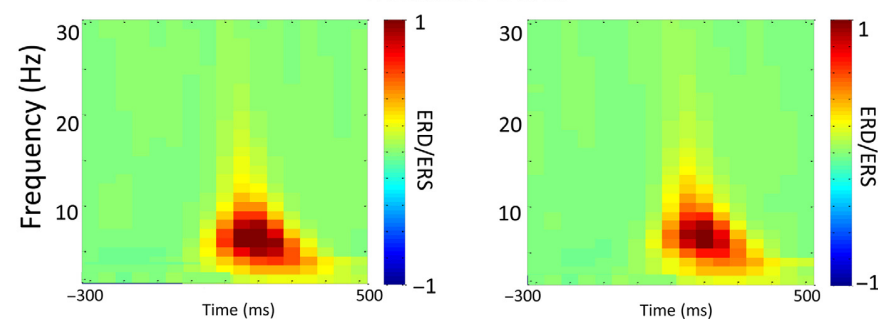

FIG. 2. Graphical representations of ERPs, Whole Power and Evoked Power recorded over the EBA cluster (PO8, P8, PO7 and P7) for all the stimuli levels. (A) Finger/Leaf; (B) Hand/Leaf Cluster; (C) Arm/Branch; (D) Body/Tree. For statistical difference between conditions, see Figs 3-5. 


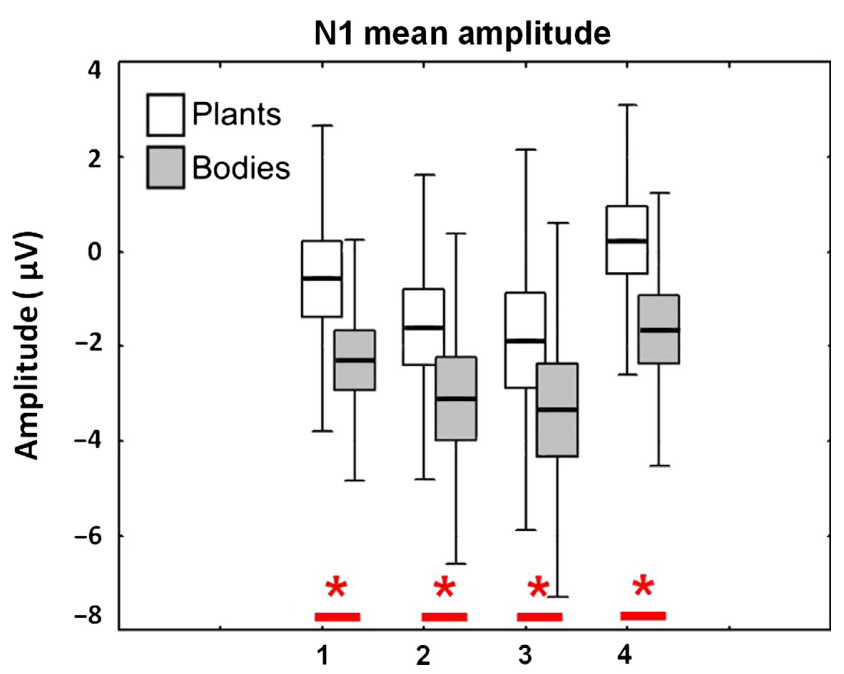

FIG. 3. N1 mean amplitudes in the different experimental conditions recorded form the cluster of electrodes over the EBA (PO7, PO8, P7, P8). Horizontal bars indicate the means, boxes indicate standard error means, and vertical bars indicate the standard deviation. Asterisks indicate significant post hoc comparisons. $1=$ Finger/Leaf; $2=$ Hand/Leaf Cluster; $3=$ Arm/ Branch; 4 = Body/Tree.

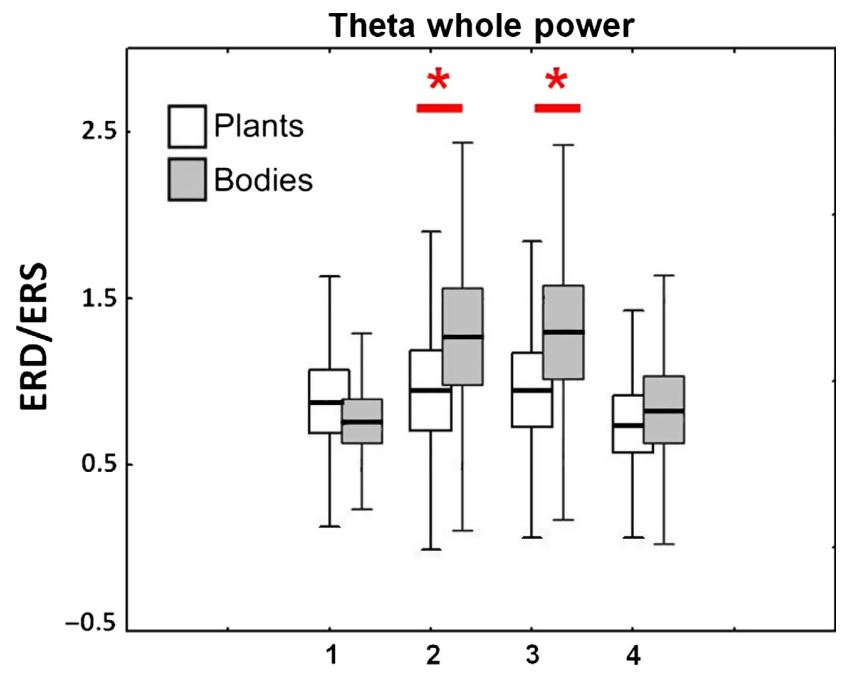

FIG. 4. The graphs show the Whole Power theta ERS over the EBA cluster for the different experimental conditions. Horizontal bars indicate the means, boxes indicate standard error means, and vertical bars indicate the standard deviation. Asterisks indicate significant post hoc comparisons. $1=$ Finger/ Leaf; 2 = Hand/Leaf Cluster; 3 = Arm/Branch; 4 = Body/Tree.

\section{Induced power}

Alpha $(8-13 \mathrm{~Hz})$ on EBA cluster. The $2 \times 4$ ANOva with Category (Body vs. Plants) $\times$ Hierarchy (finger/leaf, hand/leaf cluster, arm/ branch, body/tree) revealed a significant second level Category $\times$ Hierarchy interaction $\left(F_{3,45}=6.061, P=0.026\right)$. Post hoc tests indicated that the body images induced larger alpha ERD than hands $(P=0.021)$ (all other $P \mathrm{~s}>0.082$ ).

Theta (4-7 Hz) on EBA cluster. The $2 \times 4$ ANova with Category (Body vs. Plants) $\times$ Hierarchy (finger/leaf, hand/leaf cluster, arm/ branch, body/tree) revealed neither significant main effect nor interaction (all $P \mathrm{~s}>0.058)$.

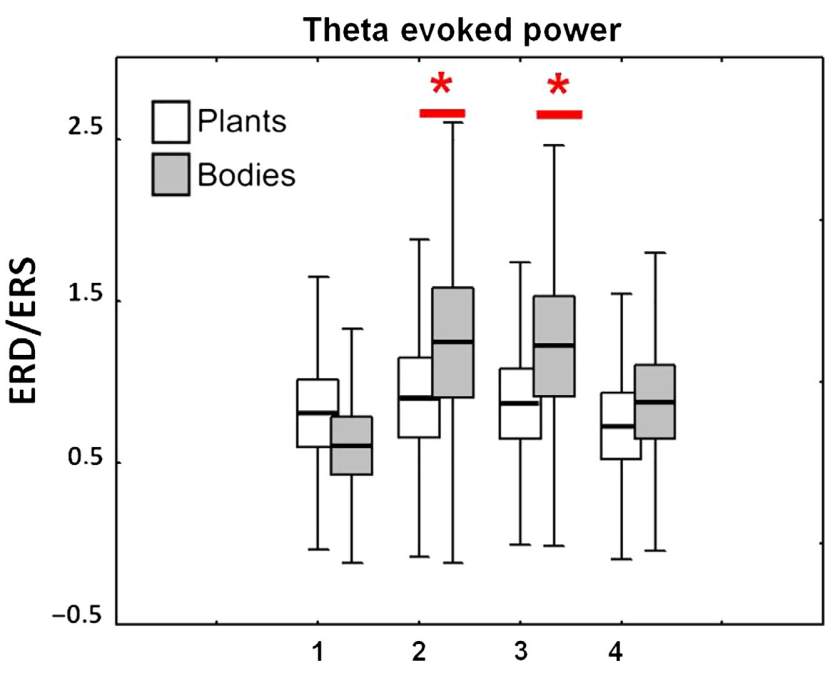

FIG. 5. The graphs show the Evoked Power theta ERS over the EBA cluster for the different experimental conditions. Horizontal bars indicate the means, boxes indicate standard error means, and vertical bars indicate the standard deviation. Asterisks indicate significant post hoc comparisons. 1 = Finger/ Leaf; 2 = Hand/Leaf Cluster; 3 = Arm/Branch; 4 = Body/Tree.

\section{Discussion}

In this study, we replicated previous findings concerning the modulation of the earliest body-selective ERP (N1) amplitude during body part and body image observation (Thierry et al., 2006; Pourtois et al., 2007; Taylor et al., 2010). We were also able to deepen the understanding of body part visual processing by: (1) highlighting a selective theta ERS for hands and arms compared to other body stimuli (i.e. fingers and full headless bodies) and to hierarchically matched control stimuli, and (2) showing that this theta modulation is due to phase-locked signal (i.e. Evoked theta power).

Thus, body and body-part stimuli seem to generate specific EEG activity in both time domain (ERP) and time-frequency domain (Whole and Evoked power) over occipito-temporal cortices. However, while evoked potentials proved to be sensitive to the same extent to body parts and full body images, only hands and arms images induced greater theta synchronization in the time window of the P1-N1 complex over EBA. This pattern of results may indicate a differential role of spectral bands for the processing of body effectors according to their relevance for actionrelated networks.

\section{ERP and ERD/ERS differences in body-related image processing}

Over the last decades, diverging theories have been trying to explain the genesis of ERPs and their counterparts in the time-frequency domain. On the one hand, studies have suggested that event-related increases in ERPs amplitude correspond to increases in EEG power compared to ongoing spontaneous EEG activity (Mäkinen et al., 2005). On the other hand, it has been suggested that ERPs are the results of a phase resetting of ongoing oscillatory activity, without changes of EEG power (Makeig et al., 2002; see Sauseng et al., 2007 for a review). It has also been suggested that both mechanisms may participate in the generation of ERP components (Herrmann et al., 2014). Concerning early visual potentials (i.e. P1-N1), the contribution of alpha and theta oscillations is considered of particular interest given the frequency of the ERP (Klimesch et al., 2004), 
but the link between time domain (ERPs) modulation and time-frequencies modulation remains unclear and needs further testing (Mazaheri \& Jensen, 2006; Sauseng et al., 2007).

In this study, while N190 amplitude over occipito-temporal electrodes was significantly higher for all body-related stimuli compared to control images, the theta ERS modulation did not reflect a similar pattern and showed a hand and arm selectivity. The Evoked power results showed a similar pattern of the Whole power theta ERS (higher for hands and arms), whereas no significant results were found in the alpha band.

Overall, although the debate on the contribution of ERD/ERS and phase-locked signal to ERP generation and modulation is still open (Mazaheri \& Jensen, 2006; Sauseng et al., 2007), the pattern of results from our study seems to be difficult to reconcile with the idea that the early ERP found for body image perception is a direct reflection of ERD/ERS in alpha and theta bands. Taken together, this evidence is in line with the notion that the ERP and the timefrequency indexes may underpin different computational mechanisms (Pfurtscheller \& Lopes da Silva, 1999; Wang, 2010). One possible explanation for these differences (see below) is that, while ERP amplitude may be more sensitive to category-specific processes (i.e. body vs. non-body), time-frequency indexes may be more sensitive to interareal communication mechanisms coding the functional role of specific visual stimuli (e.g. effectors vs. non effectors). In our case, theta modulation of the Whole and Evoked power shows a significant difference for body effectors such as hands and arms when compared to other within-category and across-category stimuli, in line with previous imaging studies that show a strong selectivity for body effectors in the occipito-temporal cortex (Bracci et al., 2012; Lingnau \& Downing, 2015).

\section{Whole bodies and body parts in the occipito-temporal cortex}

The body is considered a special object for its relevance in many cognitive functions such as self-awareness, motor control and social communication. Accordingly, the brain represents body structure and functions in many different subcortical and occipito-temporoparieto-frontal systems (Berlucchi \& Aglioti, 1997, 2010). When it concerns the visual representation of bodies, two main hypotheses have been tested in previous studies to understand whether the body is represented as a whole or as the sum of its parts in the occipitotemporal cortex. At a behavioural level, identifying and discriminating body image in inverted orientation bring about an increase in reaction times and worse accuracy, leading to the idea that full body images undergo a configural processing as for the case of faces (Seitz, 2002; Reed et al., 2003, 2006; Minnebusch \& Daum, 2009; Neri, 2009; Ramm et al., 2010; Robbins \& Coltheart, 2012; Papeo et al., 2017). Conversely, neurophysiological evidence in monkeys (Desimone et al., 1984; Bell et al., 2009; Pinsk et al., 2009; Popivanov et al., 2012) and humans (Bracci et al., 2010; Chan et al., 2010; Op de Beeck et al., 2010; Orlov et al., 2010; Weiner \& GrillSpector, 2010, 2011) suggest that different regions of the occipitotemporal cortex respond to different body parts. By testing the role of holistic vs. part-based processing of body images, a recent fMRI study showed that the EBA activity is more sensitive to the global configuration of body parts when arranged in a full body rather than when divided into single, disjointed body parts (Brandman \& Yovel, 2016). However, when testing the body configuration effect on the subclusters of EBA voxels selectively responding to single body parts, the authors of this study reported that the activity in these subclusters was not modulated by the presence of the configural body shape. The authors therefore propose that EBA contains smaller subregions coding for single body parts (as in Bracci et al., 2010; Orlov et al., 2010).

Our data seem to indicate that, although ERPs are adequate to identify neural responses selective for the content of the body shape (Thierry et al., 2006) and body parts (Taylor et al., 2007), time-frequency indices like Whole power, and more specifically Evoked power, might add information concerning the different processing mechanisms associated to different body districts.

\section{Categorical or functional representations of body-part images in the occipito-temporal cortex - the contribution of time- frequency evidence}

A different theoretical question concerns what is (are) the organizational principle(s) of the segregated representations of different body parts in the occipito-temporal cortex, and whether frequency-specific modulations might help understanding the different communication mechanisms supporting these segregated representations.

In an attempt to understand the nature of the visual responses to body images, a seminal imaging study provided initial evidence that EBA activity was also influenced by the execution of unseen armhand movements (Astafiev et al., 2004). Building on this evidence, Orlov et al. (2010) extended this idea by showing that the occipitotemporal cortex contains segregated regions responding to the visual presentation of different body parts (hands, arms, torsos, legs, feet) and that these regions also seem to somatotopically modulate their activity in response to own body-part movements. The relationship between movements and visual responses to body parts in the occipito-temporal cortex was also tested by studying the overlap between fMRI responses to hand images and tools (Bracci et al., 2012). The authors reported that responses to tools selectively overlapped with responses to hands but not with responses to whole bodies, non-hand body parts, other objects or visual motion. They noted that actionrelated object properties shared by hands effectors and tools might be reflected in the organization of high-order visual cortex (Lingnau \& Downing, 2015). The functional organization of high-order visual cortices therefore seems to partly reflect the connectivity of the visual regions with other downstream functional networks such as the fronto-parietal action network. Coherently with this proposal, imaging studies have provided evidence that seeing body effectors such as hands (Bracci et al., 2010, 2012), arms and legs (Bracci et al., 2015) activate segregated regions in the lateral occipito-temporal cortex.

Whether the visual organization of body parts in the occipito-temporal cortex is organized according to shape similarity, physical proximity, cortical homunculus proximity, or a functional principle was directly tested recently (Bracci et al., 2015). Importantly, while physical similarity accounted for fMRI responses in the occipital cortex, the largest variance was accounted for by functional similarity in occipito-temporal, and parieto-frontal, areas (Bracci et al., 2015). This evidence is compatible with the notion that anatomical connectivity of the different regions of the occipito-temporal cortex may play an important role in shaping the specificity and organization of these regions such that, once information is extracted from the visual input, it propagates to other areas where the information is further processed, integrated with information from other modalities and linked to previous experiences (Peelen \& Caramazza, 2010; Kravitz et al., 2013). Therefore, within the occipito-temporal cortex, visual information concerning the shape and motion of the body seems to converge in middle-superior temporal regions and, from here, be projected to parietal and frontal areas to build a temporoparietal-frontal network where action-related representations are processed (Lingnau \& Downing, 2015). 
It is worth noting that time-frequency modulation is thought to be associated with intra-areal as well as interareal processing. Finding theta specific modulations for hand and arm images only (not finger, full bodies or control non-body images) might represent a frequency-specific marker indicating that these body parts are further processed in action-related, temporo-parieto-frontal, networks (Lingnau \& Downing, 2015) according to their functional/semantic meaning (Bracci et al., 2015).

\section{Role of theta band in memory and perception}

Spontaneous theta frequency band oscillations were first described in the hippocampus of rabbits by Saul \& Davis (1933) and later by Green \& Arduini (1954) and have been considered to be the fingerprint of all limbic structures being most prominent in hippocampal formation (Lopes da Silva et al., 1990; Lopes da Silva, 1992). Cortical oscillations in the theta band recorded over medial-frontal sites have been related to individuals' action monitoring, cognitive control and reinforcement learning (Trujillo \& Allen, 2007; Cavanagh et al., 2009) as well as in coding motor errors observed in others (van Elk et al., 2012; Pavone et al., 2016). Most of the literature on scalp theta oscillations has dealt with activity recorded at frontal sites even when testing the neural responses to visual stimuli (for reviews see Güntekin \& Başar, 2014; Klimesch, 1999) but did not focus on early time windows ( $200 \mathrm{~ms}$ ) after stimulus presentation.

The specific appearance of theta modulations over occipito-temporal regions concerning perceptual processing is less clear as no study has so far contrasted theta responses to stimuli of different contents. Reviewing perceptual tasks, Schacter (1977) has described that occipital theta power increases (while alpha decreases) along with the complexity of visual stimulation (e.g. number of white squares, Gale et al., 1971a,b) and this evidence was interpreted in terms of the 'arousal value' of the stimulus where more arousing stimuli would yield increased theta abundance. A MEG study (Osipova et al., 2006) on a memory task using visual stimuli (buildings and landscapes) that required an encoding (content discrimination task) and a retrieval (old/new discrimination) phase reported that, during the encoding phase (similar to our task), theta ERS over right parieto-temporal region increased for later remembered items compared to forgotten ones. Here, we studied whether it was possible to detect a greater theta synchronization over electrodes that are spatially congruent with the localization of activity in visual areas specifically engaged in body-part images processing and when the associated ERP amplitude is modulated. The results seem to indicate that perceptual processes might be strengthened by mnemonic functions in a spatially organized fashion according to the specific content of the visual stimulus.

\section{Conclusions}

In this study, we tested the hypothesis that perception of bodyrelated images (compared to non-body control stimuli matched for hierarchical level) would trigger selective EEG responses in the time domain (ERPs, confirming previous studies) and in the time-frequency domain (Whole and Evoked Power) over occipito-temporal regions (Extrastriate Body Area, EBA). We were able to confirm the EBA body-specific ERP amplitude modulation (body-N190). We have extended the understanding of EBA activity during body-part perception by describing a theta synchronization increase for hand and arm images compared to other body-part (finger) and full body images (and control stimuli). Future studies will need to characterize these time-frequency responses at a functional level.

\section{Acknowledgement}

MC was supported by Sapienza University (Progetti Medi 2016). SMA was funded by PRIN grant (Progetti di Ricerca di Rilevante Interesse Nazionale, Edit. 2015, Prot. 20159CZFJK) and from H2020-SESAR-2015-1 (MOTO The embodied reMOte Tower, Project Number 699379).

\section{Conflict of interest}

The authors certify that they have no affiliations with or involvement in any organization or entity with any financial interest in the subject matter or materials discussed in this manuscript. BrainTrends did not have any financial or scientific influence on the present study. No conflicts of interest, financial or otherwise, are declared by the authors.

\section{Authors contribution}

MC and EFP designed the study. QM recorded and analysed the data. QM, EFP, SMA and MC discussed the result. QM and MC wrote the manuscript. The manuscript was edited by a native English speaker, professional proof reader.

\section{Data accessibility}

The present data will be made publicly available upon request and are stored at the Department of Psychology, Sapienza University, Rome.

\section{References}

Astafiev, S.V., Stanley, C.M., Shulman, G.L. \& Corbetta, M. (2004) Extrastriate body area in human occipital cortex responds to the performance of motor actions. Nat. Neurosci., 7, 542-548.

Bell, A.H., Hadj-Bouziane, F., Frihauf, J.B., Tootell, R.B. \& Ungerleider, L.G. (2009) Object representations in the temporal cortex of monkeys and humans as revealed by functional magnetic resonance imaging. J. Neurophysiol., 101, 688-700.

Berlucchi, G. \& Aglioti, S.M. (1997) The body in the brain: neural bases of corporeal awareness. Trends Neurosci., 20, 560-564.

Berlucchi, G. \& Aglioti, S.M. (2010) The body in the brain revisited. Exp. Brain Res., 200, 25-35.

Bracci, S. \& Peelen, M.V. (2013) Body and object effectors: the organization of object representations in high-level visual cortex reflects body-object interactions. J. Neurosci., 33, 18247-18258.

Bracci, S., Ietswaart, M., Peelen, M.V. \& Cavina-Pratesi, C. (2010) Dissociable neural responses to hands and non-hand body parts in human left extrastriate visual cortex. J. Neurophysiol., 103, 3389-3397.

Bracci, S., Cavina-Pratesi, C., Ietswaart, M., Caramazza, A. \& Peelen, M.V (2012) Closely overlapping responses to tools and hands in left lateral occipitotemporal cortex. J. Neurophysiol., 107, 1443-1456.

Bracci, S., Caramazza, A. \& Peelen, M.V. (2015) Representational similarity of body parts in human occipitotemporal cortex. J. Neurosci., 35, 1297712985.

Brandman, T. \& Yovel, G. (2016) Bodies are represented as wholes rather than their sum of parts in the occipital-temporal cortex. Cereb. Cortex, 26, 530-543.

Buzsaki, G. \& Draguhn, A. (2004) Neuronal oscillations in cortical networks. Science, 304, 1926-1929.

Cavanagh, J.F., Cohen, M.X. \& Allen, J.J. (2009) Prelude to and resolution of an error: EEG phase synchrony reveals cognitive control dynamics during action monitoring. J. Neurosci., 29, 98-105.

Cavanagh, J.F., Zambrano-Vazquez, L. \& Allen, J.J. (2012) Theta lingua franca: a common mid-frontal substrate for action monitoring processes. Psychophysiology, 49, 220-238.

Chan, A.W.-Y., Kravitz, D.J., Truong, S., Arizpe, J. \& Baker, C.I. (2010) Cortical representations of bodies and faces are strongest in commonly experienced configurations. Nat. Neurosci., 13, 417-418.

Delorme, A. \& Makeig, S. (2004) EEGLAB: an open source toolbox for analysis of single-trial EEG dynamics including independent component analysis. J. Neurosci. Meth., 134, 9-21. 
Desimone, R., Albright, T.D., Gross, C.G. \& Bruce, C. (1984) Stimulusselective properties of inferior temporal neurons in the macaque. J. Neurosci., 4, 2051-2062.

Downing, P.E., Jiang, Y., Shuman, M. \& Kanwisher, N. (2001) A cortical area selective for visual processing of the human body. Science, 293, 2470-2473.

van Elk, M., Bousardt, R., Bekkering, H. \& van Schie, H.T. (2012) Using goal- and grip-related information for understanding the correctness of other's actions: an ERP study. PLoS One, 7, e36450.

Fries, P. (2009) Neuronal gamma-band synchronization as a fundamental process in cortical computation. Аnпи. Rev. Neurosci., 32, 209-224.

Fries, P. (2015) Rhythms for cognition: communication through coherence Neuron, 88, 220-235.

Gale, A., Christie, B. \& Penfold, V. (1971a) Stimulus complexity and the occipital EEG. Brit. J. Psychol., 62, 527-531.

Gale, A., Coles, M. \& Boyd, E. (1971b) Variations in visual input and the occipital EEG: II. Psychon Sci., 23, 99-100.

Giabbiconi, C.M., Jurilj, V., Gruber, T. \& Vocks, S. (2016) Steady-state visually evoked potential correlates of human body perception. Exp. Brain Res., 234, 3133-3143.

Green, J.D. \& Arduini, A.A. (1954) Hippocampal electrical activity in arousal. J. Neurophysiol., 17, 533-557.

Gruber, W.R., Klimesch, W., Sauseng, P. \& Doppelmayr, M. (2005) Alpha phase synchronization predicts $\mathrm{P} 1$ and $\mathrm{N} 1$ latency and amplitude size. Cereb. Cortex, 15, 371-377.

Güntekin, B. \& Başar, E. (2014) A review of brain oscillations in perception of faces and emotional pictures. Neuropsychologia, 58, 33-51.

Hajihosseini, A. \& Holroyd, C.B. (2013) Frontal midline theta and N200 amplitude reflect complementary information about expectancy and outcome evaluation. Psychophysiology, 50, 550-562.

Herrmann, C.S., Rach, S., Vosskuhl, J. \& Strüber, D. (2014) Time-frequency analysis of event-related potentials: a brief tutorial. Brain Topogr., 27, 438-450.

Jung, T.-P., Makeig, S., Westerfield, M., Townsend, J., Courchesne, E. \& Sejnowski, T.J. (2000) Removal of eye activity artifacts from visual eventrelated potentials in normal and clinical subjects. Clin. Neurophysiol., 111, $1745-1758$.

Keselman, H.J. \& Rogan, J.C. (1980) Repeated measures $F$ tests and psychophysiological research: controlling the number of false positives. Psychophysiology, 17, 499-503.

Klimesch, W. (1999) EEG alpha and theta oscillations reflect cognitive and memory performance: a review and analysis. Brain Res. Rev., 29, 169-195.

Klimesch, W., Schack, B., Schabus, M., Doppelmayr, M., Gruber, W. \& Sauseng, P. (2004) Phase-locked alpha and theta oscillations generate the $\mathrm{P} 1-\mathrm{N} 1$ complex and are related to memory performance. Cognitive Brain Res., 19, 302-316.

Kravitz, D.J., Saleem, K.S., Baker, C.I., Ungerleider, L.G. \& Mishkin, M. (2013) The ventral visual pathway: an expanded neural framework for the processing of object quality. Trends Cogn. Sci., 17, 26-49.

Lingnau, A. \& Downing, P.E. (2015) The lateral occipitotemporal cortex in action. Trends Cogn. Sci., 19, 268-277.

Lopes da Silva, F.H. (1992). The rhythmic slow activity (theta) of the limbic cortex: an oscillation in search of a function. In Basar, E. \& Bullock, T.H. (Eds), Induced Rhythms in the Brain. Birkhauser, Boston, pp. 269-283.

Lopes da Silva, F.H., Witter, M.P., Boeijinga, P.H. \& Lohman, A.H. (1990) Anatomic organization and physiology of the limbic cortex. Physiol. Rev., 70, 453-511.

Makeig, S., Westerfield, M., Jung, T.-P., Enghoff, S., Townsend, J., Courchesne, E. \& Sejnowski, T.J. (2002) Dynamic brain sources of visual evoked responses. Science, 295, 690-694.

Mäkinen, V., Tiitinen, H. \& May, P. (2005) Auditory event-related responses are generated independently of ongoing brain activity. Neuroimage, 24, 961-968.

Mazaheri, A. \& Jensen, O. (2006) Posterior alpha activity is not phase-reset by visual stimuli. Proc. Natl. Acad. Sci. USA, 103, 2948-2952.

Minnebusch, D.A. \& Daum, I. (2009) Neuropsychological mechanisms of visual face and body perception. Neurosci. Biobehav. R., 33, 1133-1144.

Moro, V., Urgesi, C., Pernigo, S., Lanteri, P., Pazzaglia, M. \& Aglioti, S.M. (2008) The neural basis of body form and body action agnosia. Neuron, 60, 235-246.

Neri, P. (2009) Nonlinear characterization of a simple process in human vision. J. Vision, 9, 1-29.
Oostenveld, R., Fries, P., Maris, E. \& Schoffelen, J.-M. (2010) FieldTrip: open source software for advanced analysis of MEG, EEG and invasive electrophysiological data. Comput. Intel. Neurosc., 2011, 156869.

Op de Beeck, H.P., Brants, M., Baeck, A. \& Wagemans, J. (2010) Distributed subordinate specificity for bodies, faces, and buildings in human ventral visual cortex. Neuroimage, 49, 3414-3425.

Orlov, T., Makin, T.R. \& Zohary, E. (2010) Topographic representation of the human body in the occipitotemporal cortex. Neuron, 68, 586600 .

Osipova, D., Takashima, A., Oostenveld, R., Fernández, G., Maris, E. \& Jensen, O. (2006) Theta and gamma oscillations predict encoding and retrieval of declarative memory. J. Neurosci., 26, 7523-7531.

Papeo, L., Stein, T. \& Soto-Faraco, S. (2017) The two-body inversion effect. Psychol. Sci., 28, 369-379.

Pavone, E.F., Tieri, G., Rizza, G., Tidoni, E., Grisoni, L. \& Aglioti, S.M. (2016) Embodying others in immersive virtual reality: electro-cortical signatures of monitoring the errors in the actions of an avatar seen from a first-person perspective. J. Neurosci., 36, 268-279.

Peelen, M.V. \& Caramazza, A. (2010) What body parts reveal about: the organization of the brain. Neuron, 68, 331-333.

Peelen, M.V. \& Downing, P.E. (2005) Selectivity for the human body in the fusiform gyrus. J. Neurophysiol., 93, 603-608.

Pfurtscheller, G. (2001) Functional brain imaging based on ERD/ERS. Vision. Res., 41, 1257-1260.

Pfurtscheller, G. \& Lopes da Silva, F.H. (1999) Event-related EEG/MEG synchronization and desynchronization: basic principles. Clin. Neurophysiol., 110, 1842-1857.

Pinsk, M.A., Arcaro, M., Weiner, K.S., Kalkus, J.F., Inati, S.J., Gross, C.G. \& Kastner, S. (2009) Neural representations of faces and body parts in macaque and human cortex: a comparative FMRI study. J. Neurophysiol., 101, 2581-2600.

Pitcher, D., Charles, L., Devlin, J.T., Walsh, V. \& Duchaine, B. (2009) Triple dissociation of faces, bodies, and objects in extrastriate cortex. Curr. Biol., 19, 319-324.

Pitcher, D., Goldhaber, T., Duchaine, B., Walsh, V. \& Kanwisher, N. (2012) Two critical and functionally distinct stages of face and body perception. J. Neurosci., 32, 15877-15885.

Popivanov, I.D., Jastorff, J., Vanduffel, W. \& Vogels, R. (2012) Heterogeneous single-unit selectivity in an fMRI-defined body-selective patch. $J$. Neurosci., 34, 95-111.

Pourtois, G., Peelen, M.V., Spinelli, L., Seeck, M. \& Vuilleumier, P. (2007) Direct intracranial recording of body-selective responses in human extrastriate visual cortex. Neuropsychologia, 45, 2621-2625.

Ramm, B.J., Cummins, T.D.R. \& Slaughter, V. (2010) Specifying the human body configuration. Vis. Cogn., 18, 898-919.

Reed, C.L., Stone, V.E., Bozova, S. \& Tanaka, J. (2003) The body-inversion effect. Psychol. Sci., 14, 302-308.

Reed, C.L., Grubb, J.D. \& Steele, C. (2006) Hands up; attentional prioritization of space near the hands. J. Exp. Psychol. Human, 21, $166-177$

Robbins, R. \& Coltheart, M. (2012) Left-right holistic integration of human bodies. Q. J. Exp. Psychol., 65, 1962-1974.

Saul, L.J. \& Davis, H. (1933) Action currents in the central nervous system. Arch. Neurol. Psy., 29, 255-259.

Sauseng, P., Klimesch, W., Gruber, W.R., Hanslmayr, S., Freunberger, R. \& Doppelmayr, M. (2007) Are event-related potential components generated by phase resetting of brain oscillations? A critical discussion. Neuroscience, 146, 1435-1444.

Schacter, D.L. (1977) EEG theta waves and psychological phenomena: a review and analysis. Biol. Psychol., 5, 47-82.

Schwarzlose, R.F., Baker, C.I. \& Kanwisher, N. (2005) Separate face and body selectivity on the fusiform gyrus. J. Neurosci., 25, 11055-11059.

Seitz, K. (2002) Parts and wholes in person recognition: developmental trends. J. Exp. Child Psychol., 82, 367-381.

Taylor, J.C., Wiggett, A.J. \& Downing, P.E. (2007) Functional MRI analysis of body and body part representations in the extrastriate and fusiform body areas. J. Neurophysiol., 98, 1626-1633.

Taylor, J.C., Roberts, M.V., Downing, P.E. \& Thierry, G. (2010) Functional characterisation of the extrastriate body area based on the N1 ERP component. Brain Cognition, 73, 153-159.

Thierry, G., Pegna, A.J., Dodds, C., Roberts, M., Basan, S. \& Downing, P. (2006) An event-related potential component sensitive to images of the human body. Neuroimage, 32, 871-879. 
Trujillo, L.T. \& Allen, J.J. (2007) Theta EEG dynamics of the error-related negativity. Clin. Neurophysiol., 118, 645-668.

Urgesi, C., Berlucchi, G. \& Aglioti, S.M. (2004) Magnetic stimulation of extrastriate body area impairs visual processing of nonfacial body parts. Curr. Biol., 14, 2130-2134.

Urgesi, C., Candidi, M., Ionta, S. \& Aglioti, S.M. (2007) Representation of body identity and body actions in extrastriate body area and ventral premotor cortex. Nat. Neurosci., 10, 30-31.

Wang, X.-J. (2010) Neurophysiological and computational principles of cortical rhythms in cognition. Physiol. Rev., 90, 1195-1268.
Weiner, K.S. \& Grill-Spector, K. (2010) Sparsely-distributed organization of face and limb activations in human ventral temporal cortex. Neuroimage, 52, 1559-1573.

Weiner, K.S. \& Grill-Spector, K. (2011) Not one extrastriate body area: using anatomical landmarks, hMT+, and visual field maps to parcellate limb-selective activations in human lateral occipitotemporal cortex. Neuroimage, 56, 2183-2199. 